\title{
THE EVOLUTION OF MODELLING PRACTICES ON CANADA'S PARLIAMENT HILL:AN ANALYSIS OF THREE SIGNIFICANT HERITAGE BUILDING INFORMATION MODELS (HBIM)
}

\author{
L. Chow ${ }^{1}$, K. Graham ${ }^{1}$, T. Grunt ${ }^{1}$, M.Gallant ${ }^{1}$, J. Rafeiro, ${ }^{1}$ S. Fai ${ }^{1} *$ \\ ${ }^{1}$ Carleton Immersive Media Studio (CIMS), Carleton University, Ottawa, Canada - (lchow, kgraham, tgrunt, mgallant, \\ jraferio,sfai)@cims.carleton.ca
}

KEY WORDS: Heritage Building Information Model, Level of Detail, Model Tolerance, Level of Accuracy, Data management

\begin{abstract}
:
In this paper, we explore the evolution of modelling practices used to develop three significant Heritage Building Information Models (HBIM) on Canada's Parliament Hill National Historic Site - West Block, Centre Block, and The Library of Parliament. The unique scope, objective, and timeline for each model required an in-depth analysis to select the appropriate classification for Level of Detail (LOD) and Level of Accuracy (LOA). With each project, the refinement of modelling practices and workflows evolved, culminating in one of our most complex and challenging projects - the Library of Parliament BIM. The purpose of this paper is to share ideas and lessons learned for the intricate challenges that emerge when using LOD and LOA classifications including trade-offs between model performance, tolerances, and anticipated BIM use. In addition, we will evaluate how these decisions effected managing the digitization, data processing, data synthesis, and visualisation of the models.
\end{abstract}

\section{OVERVIEW}

In 2012, Public Services and Procurement Canada (PSPC) and the Carleton Immersive Media Studio (CIMS) began a research partnership to explore the application of digital technologies for architectural rehabilitation and heritage conservation. Our research has focused on the Parliament Buildings National Historic Site of Canada and has explored: methodologies for digitization (integrating photogrammetry and terrestrial laser scanning); building information modelling of historic structures (HBIM); digitally assisted fabrication (robotic milling and 3D printing); and digitally assisted storytelling (web, mobile, and virtual and augmented reality).

In this paper, we focus on the evolution of our BIM practices on Parliament Hill stemming from the research initiative with PSPC, and more specifically, address the challenges in selecting the appropriate Level of Detail (LOD), and Level of Accuracy (LOA) / model tolerance. Establishing an appropriate LOD and LOA is a crucial decision in defining the scope of a BIM project as it has a significant impact on model use, efficiency, and management. However, the complex geometry and deformations often found in existing and historical buildings make it difficult to adopt existing BIM standards that have been developed for new construction. Our study will revolve around three of the four heritage buildings situated on the Hill - West Block, Centre Block, and the Library of Parliament. A detailed analysis of the scope of the project, data management practices, and modelling methodology will demonstrate an evolution of modelling practices and workflows leading to best practices and lessons learned.

\subsection{Canada's Parliament Hill National Historic Site}

The Parliament Hill National Historic Site of Canada is comprised of the Centre Block, East Block, West Block, and Library of Parliament and is Canada's most recognized national monument. As both the political and symbolic locus of Canada's parliamentary democracy, the site is in every sense a stage where Canada's nationhood is played out for national and international audiences.

Construction of the Parliament Buildings began in 1859, and in 1866 , they were officially opened to the public. In 1916, tragedy struck when the original Centre Block building was destroyed by fire. Reconstruction of Centre Block began immediately with the new design developed by the architects John A. Pearson and Jean-Omer Marchand. The first sitting of Parliament in the new building occurred four years later, but it was not until 1927 that the 98 metre (320 foot) Peace Tower was completed. Today, the Parliament Hill National Historic site is admired for its exemplary Gothic Revival style. Both the grounds and buildings are recognized for their heritage significance and have been designated as Federal Heritage Buildings (FBHRO).

A comprehensive rehabilitation program for Parliament Hill commenced in 2002 - beginning with the Library of Parliament - with the intention of repairing the historic fabric, modernizing services, and addressing changes in the functional program. Following the Library, the rehabilitation of West Block began in 2011 and was completed in late 2018. The Centre Block program of work is now underway. The East Block will see two phases of rehabilitation - the first beginning in 2017 and the second phase in the near future.

\section{CIMS LODIA}

\subsection{Description}

During the development of the West Block BIM (2013) and the initial stages of the Centre Block BIM (2015), LOD and LOA standards or guidelines for modelling heritage buildings did not exist. In order to establish consistency in our own work, CIMS began the development of internal guidelines in 2015. The three-

\footnotetext{
* Corresponding author
} 
tier, five category level of development system borrows from the architecture, engineering, construction and operations (AECO) industry standards and guidelines and indexes level of detail (LOD), level of information (LOI), and level of accuracy (LOA) - LODIA - for each element type.

In the acronym, $>\mathrm{D}<$ refers to the graphical and geometric data; $>\mathrm{I}<$ refers to the embedded information related to the geometry; and $>A<$ refers to accuracy and resolution. Separating LOA from LOD provides an opportunity to register differences between model geometry and sensor data that may result from having to hasten the creation or optimize the operation of the model. For the purpose of this paper, a brief review of the CIMS LODIA LOD and LOA classifications follow. For a detailed description of the CIMS LODIA, see Graham et al., 2018.

\subsection{CIMS LOD Classification System}

CIMS LOD describes graphical and geometric representation in a scale from simple placeholder to detailed model and is based on existing standards for new buildings (Figure 1). The selection of a specific level of detail is determined by available sensor data and reference material and anticipated BIM uses. The LOD breakdown is as follows: LOD 0 - the element may not be modelled and may be represented by a placeholder (e.g. point cloud, historical drawing). If modelled, the element is a generic form with nonspecific dimensions and geometry; LOD 1 - the model element shows the generic size and shape graphically but does not contain additional information such as material, detailing, etc. For example, a window is represented as an outline only. It contains proper dimensions but does not show details; LOD 2 - the model element is represented graphically with primary materials shown. Connections and secondary materials are minimally represented; LOD 3 - the model element is accurately represented graphically. The material palette is shown and connections are modelled - but fasteners are not; LOD 4 the model element represents ALL graphical and geometric information, including fasteners and the size of individual members. This LOD is reserved for areas where comprehensive detail is required.

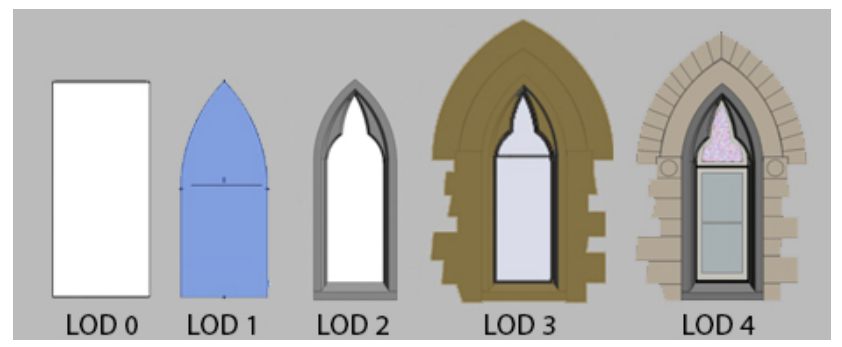

Figure 1. CIMS Level of Detail 0 to 4 of a window

\subsection{CIMS LOA Classification System}

In the context of the CIMS LODIA protocol, LOA reflects the level to which the deflection and deviation of the building element are modelled in the BIM. LOA is characterized as: LOA $0-$ no deflection/deviation is modelled. An average dimension is used for position and material thickness; LOA 1 - element deflection/deviation is modelled at corners and changes of materials. Deflection/deviation shown is in positioning and not in material thickness; LOA 2 - element deflection/deviation is modelled at a predetermined grid spacing (typically between $1000 \mathrm{~mm}$ and $3000 \mathrm{~mm}$ ) and at corners or changes of material. Deflection/deviation is shown in positioning, not in material thickness; LOA 3 - element deflection/deviation is modelled at a predetermined grid spacing (typically between $300 \mathrm{~mm}$ and $1000 \mathrm{~mm}$ ) and at corners or changes of material. Large deflections/deviations (typically $50 \mathrm{~mm}$ to $1000 \mathrm{~mm}$ ) between grid spacing are added. Deflection/deviation is shown in positioning and in material thickness if the thickness shows a deflection/deviation greater than $25 \mathrm{~mm}$; LOA 4 - the highest level of accuracy is accomplished through the creation of a mesh generated from point cloud data and contains all deflection and deformation.

\section{LOD AND LOA SPECIFICATIONS FOR HBIM}

Existing standards for new buildings have not addressed the particular challenges for BIM in the context of architectural conservation or rehabilitation. As little as four years ago, literature reviews on HBIM recognized the limited application of BIM for existing buildings (Volk et. Al, 2014). Since then, the application of and literature on BIM for historical buildings has been increasing rapidly. However, one of the fundamental problems, as outlined by several reviews, is the lack of agreedupon HBIM standards and classifications (Dore et al., 2017, Volk et al., 2014., Hichri et al., 2013). To date, only two guides have been published addressing the need for BIM specifications for historical buildings. Specifically, Historic England's BIM for Heritage (2017) and BIM for Heritage: Developing an Asset Information Model - Consultation Copy (2018), and Plowman Craven's BIM Survey Specification and Reference Guide (2015). These guides build upon existing classifications systems for LOD and LOA - developed for new construction - and augment these systems for historical and heritage BIM. The following section reviews the definitions of LOD and LOA offered within these guidelines.

\subsection{Level of Detail}

In new construction, the LOD is dependent on the specific information required for each phase of the design from conception to the realized building (Volk et al., 2014, Rafiero et al., 2013). Understandably, as the project nears completion the model becomes more detailed as more information has been obtained (BIM FORUM). In contrast, for existing and heritage buildings, a high LOD may be realized during the early phases of the project using information from survey data and secondary sources.

The Plowman Craven Reference Guide uses the British Standard Institution's (BSI) PAS 1192-2:2013 to define Level of Detail as, "Description of graphical content of models at each of the stages defined for example in the CIC Scope of Services" (Plowman Craven, 2015). The prescribed LOD for a heritage building is specified at the highest LOD, LOD4/5, which is then demonstrated through a series of tables and images for each building element.

The Historic England guide references two guides - the $A E C$ (UK) BIM Technology Protocol (AEC (UK) 2015 and Metric Survey Specifications for Cultural Heritage (Andrews et al., 2015) to demonstrate LOD. It is noted that, although the systems are meant for new construction, 'it is possible to define and communicate the level of (geometric) detail required for models, systems, assemblies or individual components" (Historic England, 2017). For heritage projects, the guide advises modelling to the minimal level of graphic detail required for the specific BIM use. (Historic England, 2017). 


\subsection{Level of Accuracy and Modelling Tolerances}

The Plowman Craven Reference Guide addresses both survey LOA and model LOA (modelling tolerances). It defines three levels of modelling tolerances measurable as the deviation from model data to point cloud: Low-Level tolerance: $60 \mathrm{~mm}$, MidLevel tolerance $30 \mathrm{~mm}$, and High-Level tolerance $15 \mathrm{~mm}$.

Although the Historic England's BIM for Heritage (2017) guide identifies categories and descriptions of varying LOD, the document only defines modelling tolerances generally and does not specify specific modelling tolerance classifications for historical buildings nor does it reference secondary sources.

\section{WEST BLOCK BIM (2013)}

\subsection{Initial Scope of Work}

In July 2013, CIMS was tasked by PSPC with developing a BIM of the West Block building using geo-referenced point cloud data. At the time, the development of BIM for historical buildings of that scale was a novel idea - the potential uses, challenges, and best practices were unknown. Therefore, the intention was to investigate the potential value of digitization and BIM technology for the documentation, rehabilitation, and long-term management of the Parliament Hill site - and beyond.

The first challenge presented itself in selecting the appropriate LOD for the model. Was it possible to reconcile the dataintensive accuracy necessary for a comprehensive heritage record with the speed necessary for a functional model? At the outset of the project, the anticipated BIM use had not been defined. Thus, there was an opportunity to analyse and compare varying levels of LOD. However, this also meant that the modelling strategy needed to remain flexible in terms of the ability to accommodate future BIM uses.

When the West Block BIM project began, CIMS adopted the only national LOD guideline available - the $A E C$ (CAN) BIM Protocol, published by the Canada BIM Council (CanBIM) in 2012. The CanBIM document followed the AEC (UK) BIM Protocol v.2 - also published in 2012. The protocol was platform independent but supplemented by software vendorspecific addenda.

In consultation with PSPC, it was initially decided to divide West Block into three separate zones - each assigned a specific LOD prescribed by the AEC CAN BIM 2013 specifications. LOD 1: South, West, and North Wings; LOD2: East Wing; LOD3: Mackenzie Tower. In addition to these three zones, CIMS developed a highly detailed area of one of the wash courses of the Mackenzie Tower to test the potential of the BIM software to support the efforts of the masons working on the rehabilitation. Specifically, could the model - developed to a $5 \mathrm{~mm}$ tolerance - be used to determine how the structure could be disassembled, repaired, and reassembled both safely and efficiently?

\subsection{Data Sources and Management}

The primary data used to develop the West Block BIM was from geo-referenced point cloud data, augmented with a diverse set of secondary data including photographs, historical drawings, 2D CAD plans, 2D CAD elevations based on orthographic photographs, and total station surveys. The interior survey was completed using the Faro Focus 3D. The exterior was surveyed using the Leica C10, with supplementary data from the Faro. The point cloud data was supported by an extensive photographic record, field notes, and hand measures taken during site inspections.

In instances where scanning was not possible, the BIM followed secondary sources such as the CAD files prepared by PSPC. To amplify the complexity of the project, the building was an active construction site, requiring several scanning campaigns to capture the building through the construction phases to completion. Due to construction, some areas were not accessible to scan - such as the stairwells, portions of the interior, and the North Façade - until after the construction was complete. In total, 1.5 terabytes of geo-referenced point cloud data were captured.

To enhance workstation performance and decrease model file size, the model was also divided into several component models that when linked together created a federated model. The component models included: roof, shell, interiors, slabs, and historic structure (Figure 2).
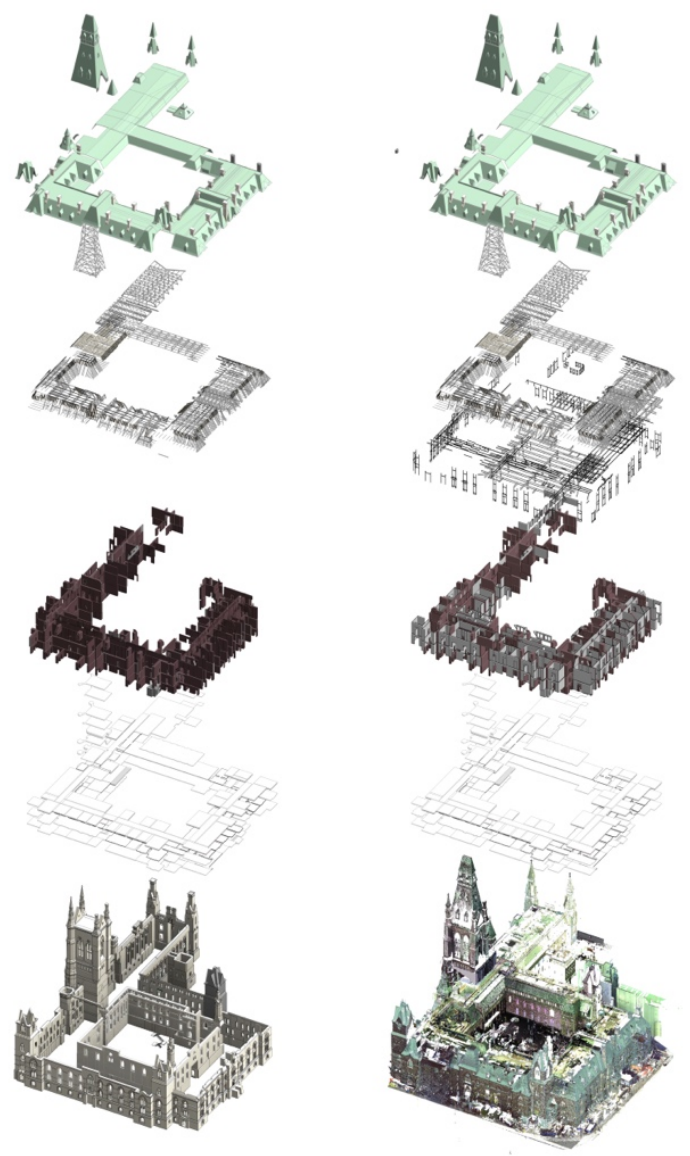

Figure 2. Component Models for West Block BIM as modelling progressed.

\subsection{Modelling Methodology}

The first step in developing the West Block BIM was to integrate the geo-referenced point cloud data into Revit 2014 by linking the individual .pts files. The point cloud data was then viewed in a series of $2 \mathrm{D}$ section, elevation, and plan views to trace the profiles of the building element geometry to the assigned LOD and LOA. Next, using Revit's modelling tools, a solid 3D model element was created. 
For LOD1, elements were modelled as a simple placeholder with a minimal level of detail. Secondary sources were relied on heavily, as most areas specified at LOD 1 were not captured in the initial set of point cloud data. For LOD2, building element types were developed from point cloud data in Revit - such as a window - and used to produce a library of parametric families. These building elements were then parametrically adjusted to accommodate their location in the model. At LOD3, building elements were meshed from sensor data to generate model-inplace elements that afforded very specific and accurate models of unique geometric characteristics.

\subsection{Results and Subsequent Scopes of Work}

The difficulty at the time of the West Block BIM project - and still today - were the limitations of BIM software for the modelling of existing conditions. Generally, the literature on projects using similar point cloud to BIM workflows have expressed similar concerns (Brumana et al., 2013, Fai et al., 2013, D'Auria et al., 2014, Jose Lopez et al., 2018). To address these limitations, CIMS developed a series of protocols - step-by-step graphic guides - to explain novel techniques for modelling from point cloud data.

In regard to LOD and efficiency, we discovered that modelling to the highest LOD capturing the majority of deformations using model in-place components — while demonstrating more geometric fidelity - was a time-consuming process and decreased the functionality of the model. Modelling at a lower LOD produced a model with a lower LOA but improved model functionality.

Another important observation regarding LOD and LOA is the quality of the survey data. During the modelling of West Block, the resolution of the point cloud data was not high enough to model at a high LOD without relying on secondary sources. Based on our experience, it is imperative that a detailed documentation strategy is undertaken prior to developing a BIM for heritage documentation, conservation, and management in order to determine and reconcile the LOD of both the survey and the model.

Following the initial phase of modelling (2013-2015), the second phase of modelling began in the summer of 2015. The scope of the project included increasing the LOD of the first phase elements and modelling all of the new retrofit for West Block, including the seismic upgrades, new subterranean levels, and the Visitor's Welcome Centre Addition. Generally, the model was developed following AEC CAN BIM LOD2.

\section{CENTRE BLOCK BIM (2015)}

\subsection{Initial Scope of Work}

After demonstrating the potential value of BIM through the West Block BIM project, PSPC tasked CIMS with producing a Model of the Centre Block in 2015. The intention of creating an existing conditions BIM of the Centre Block was to facilitate aspects of an integrated project delivery (IPD) method for the Centre Block Program of Work. CIMS would develop the BIM and hand the model over to the AEC consultant team responsible for the rehabilitation work. In addition to capturing the existing conditions of the building, the model was developed in anticipation of specific model uses including, but not limited to, the generation of drawings, site analysis, design coordination, and design authoring.
In order to meet these objectives, the appropriate LOD for each building element category required specification. Our initial proposal was to utilize a commonly accepted BIM specification classification system - The Level of Development Specification developed in the United States by BIMFORUM. The system combined level of detail with level of information classifications into level of development. A simplified level of development system was established between PSPC and CIMS for the initial scope, assigning LOD to specific building element categories: LOD 300: exterior walls, roofs, foundations, structural elements (verified to point cloud); LOD 200: interior walls, stairways, slabs, structural elements (not verified to point cloud). LOD 300 was also assigned to spaces of significant heritage value such as the Senate Chamber, House of Commons chambers, Senate and House of Commons foyers, Rotunda, and Hall of Honour.

The model tolerance for the Centre Block BIM was determined by comparing the deflection and deviation of the building element to point cloud data. It was determined that a tolerance of less than $25 \mathrm{~mm}$ of modelled elements to point cloud data was acceptable. Any deviations greater than $25 \mathrm{~mm}$ would be captured within the geometric representation of the building element. Additionally, when the deflection or deformation was determined to be worth noting - based off of the defined LOA - it was recorded in a customized properties panel. Three categories were defined: lateral deviation, vertical deviation, and irregularity of geometry. Lateral and vertical deviation referred to changes in the profile whereas irregularity of geometry referred to deviations such as missing or broken elements.

\subsection{Data Sources and Management}

The Centre Block BIM required the synthesis of large, diverse data sets. The primary data source was geo-referenced point cloud data from terrestrial laser scanning and photogrammetry. The data was captured by CIMS in partnership with HCS using a Leica $\mathrm{C} 10$ and P40 (exterior and large interior spaces) and a Faro Focus (small to mid-sized interior spaces). Significant heritage interiors including the Senate, Senate Foyer, House of Commons, House of Commons Foyer, Rotunda, Hall of Honour and the exterior of the Peace Tower were also captured by HCS using photogrammetry. Over 2000 individual scan stations were required to capture the interior and exterior of Centre Block resulting in over four terabytes of point cloud data.

Secondary sources such as archival drawings, photographs, historical steel catalogues, and technical reports were referenced in cases where point cloud data was not available. For example, the structural steel that is normally hidden from view and cannot be captured by laser scanning or photogrammetry.

To increase workstation performance while modelling, the .pts files were imported into Autodesk Recap and divided into .rcp files by areas per floor. For instance, $3^{\text {rd }}$ floor south-west, $3^{\text {rd }}$ floor south-east, $3^{\text {rd }}$ floor East Office Block, etc. This way, model users could turn on/off specific areas of point cloud through the Revit Visibility and View settings for a small, localized area instead of loading in a large data set.

Due to the size of the physical building, in an effort to minimize model file size, we developed multiple component models that when linked together created a federated model. The component models included: roof, shell, interiors, circulation and slabs, and structure (Figure 3). 


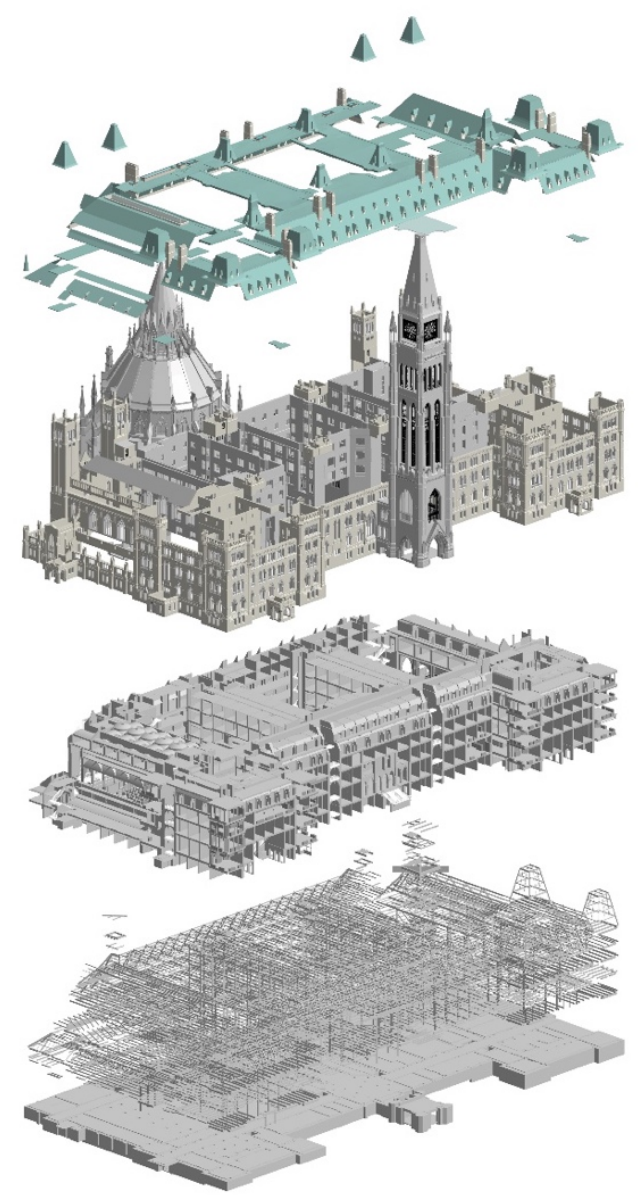

Figure 3. Exploded Axonometric of the Centre Block BIM showing the linked models.

\subsection{Modelling Methodology}

Similar in methodology to the West Block BIM, the first step in modelling Centre Block was integrating the geo-referenced point cloud data into Revit by linking the individual .rcp files. The point cloud data was then viewed in a series of $2 \mathrm{D}$ section, elevation, and plan views to trace the profiles of the building elements geometry to the assigned LOD and LOA. Next, using Revit's modelling tools a solid 3D model element was developed into parametric families.

As modelling progressed, we realized that BIMFORUM specification was insufficient for developing BIMs of historical buildings such as Centre Block. The availability of information for in-situ building elements varied significantly, creating the need to identify levels of geometric detail, non-graphical information, and accuracy. In an effort to clarify the terms of reference, CIMS proposed the use of the CIMS LODIA resulting in a more nuanced system of classification:

\begin{tabular}{|l|l|l|l|}
\hline BIM Category & LOD & LOI & LOA \\
\hline exterior geometry & 3 & 2 & 2 \\
\hline verified structural elements & 3 & 2 & 2 \\
\hline unverified structural elements & 2 & 2 & 0 \\
\hline interior spaces & 2 & 2 & 1 \\
\hline specialty spaces & 3 & 2 & 2 \\
\hline
\end{tabular}

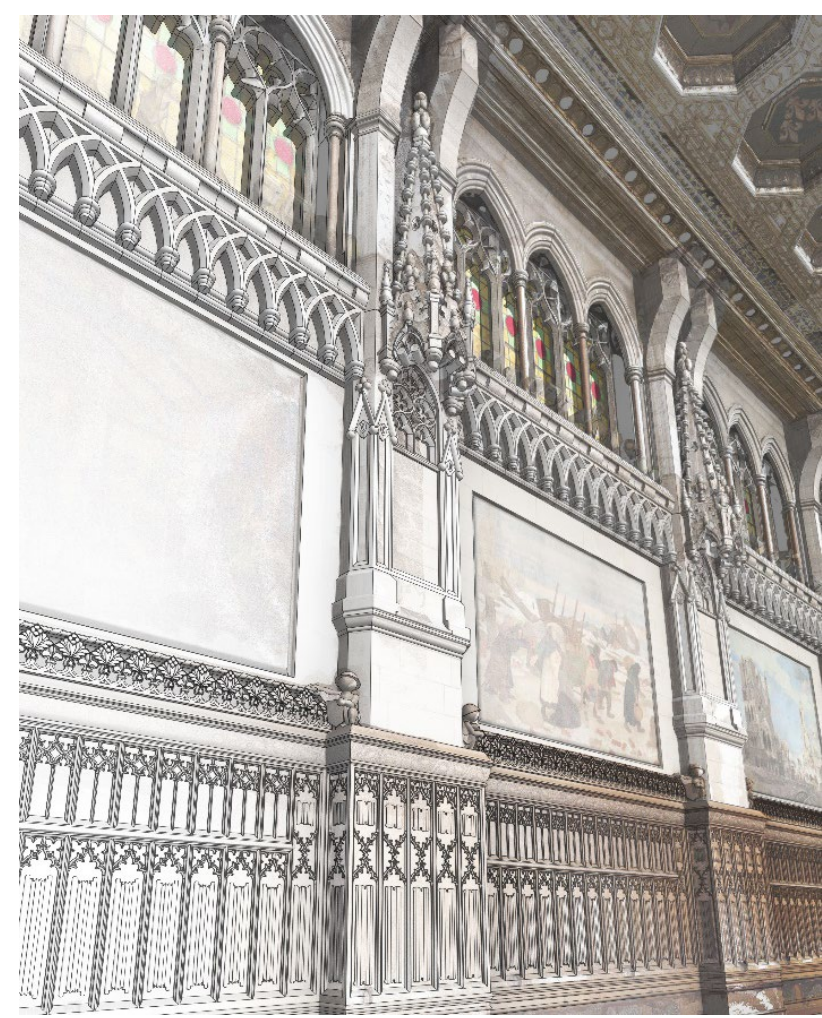

Figure 4. The Senate Chamber BIM at LOD 4

The LODIA of each component was based on an understanding of the available data from the source material, as well as the anticipated use for that data. For example, the structural steel beams within the floor slabs could be identified as LOD 2, LOI 2 , and LOA 0 . The LOD and LOI were high because of the available archival drawings and historical catalogues including standard measurements and profiles from the Bethlehem Steel Catalogues from 1906-1911. However, since there was no point cloud data available - the vast majority of the steel was behind masonry walls and not visible to remote sensors - the LOA was 0 .

In comparison, the Senate Chamber (Figure 4) exhibited a different LODIA. While all specialty rooms in the Centre Block BIM were initially targeted to be modelled at a LOD 3, the Senate Chamber increased in graphical detail to a LOD 4 and LOA 4, while LOI was reduced to 2 . This was because the model geometry of the Senate Chamber was leveraged for a virtual reality project related to the rehabilitation and required a high LOD for visualization purposes. High-resolution laser scanning and photogrammetry were used to record the space. However, very little verified information about wall assembly or materiality was available.

\subsection{Results Subsequent Scopes of Work}

In early 2017, the Centre Block BIM was handed over to the architecture and engineering consultants - CENTRUS. At the time, portions of the model were incomplete or underdeveloped due to time constraints and insufficient point cloud data. Following the hand-over, the CENTRUS team contracted CIMS to complete the existing conditions model and to research the application of emerging digital technologies for heritage conservation through additional research projects. 
Through our meetings with CENTRUS, it was evident that the LOD needed to be augmented for specific BIM uses and users. A low LOD was required for conceptual/schematic planning, making the existing model too detailed, while a high LOD was required for visualizations and heritage asset management. One approach that we explored was developing model elements to three LOD. Using the Revit coarse, medium, fine detail settings, users were able to view all three LOD within one model depending on required information or use (Figure 5). As the second phase of modelling progressed, the LOD and scoped transformed prompting another division of the models due to increased file size. Model elements were divided into additional linking files including basement, heritage, courtyards, interiors (floors 1-3), and interiors (floors 4-6).

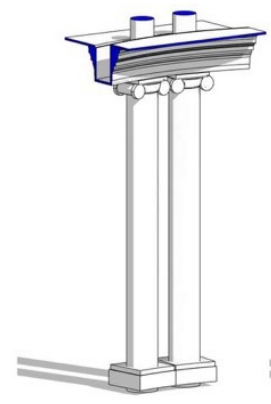

COARSE

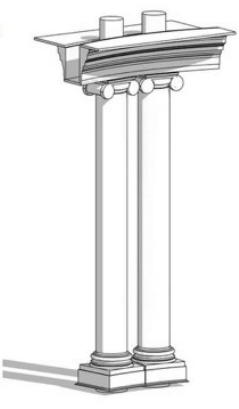

MEDIUM

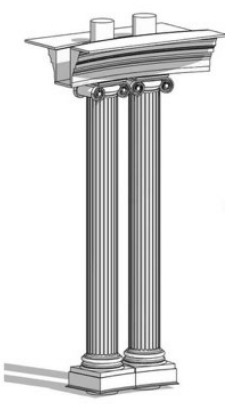

FINE
Figure 5. Column developed at three LOD for the Coarse, Medium and Fine Revit detail levels.

The interdisciplinary research taking place between members of industry (CENTRUS) and academia (CIMS) has proven to be tremendously beneficial for both groups. The exchange of innovative workflows from research and standard practices from industry has pushed the application of digital tools for heritage conservation. The initiative is also supporting the development of highly qualified personnel (HQP) preparing researchers for both the intellectual and technical demands of a leadership role in defining the role of digital tools for heritage conservation in Canada - and beyond.

\section{LIBRARY OF PARLIAMENT BIM (2017)}

\subsection{Scope of Work}

Our work on Parliament Hill culminated in the fall of 2017, with the digitization and modelling of one of Canada's most significant heritage assets - the Library of Parliament. PSPC tasked CIMS with developing a BIM of the Library in anticipation of the following BIM uses: visualization for communication, scheduling / phase planning, site utilization planning, digital and digitally assisted fabrication, heritage asset management, field Management, and record Model. As a tool for heritage asset management and visualization purposes, the model required the highest level of detail and accuracy. We returned to the 2016 Level of Development Specification published by BIMFORUM as an attempt to utilize industry standard classifications. While the specification acknowledges that it is not possible to assign a comprehensive LOD designation, the goal of the Library BIM was to model to the highest level of development possible -500 - where and when enough point cloud data was available. BIMFORUM utilizes the AIA definition of LOD500 as ".... field verified representation in terms of size, shape, location, quantity, and orientation. Nongraphic information" (BIMFORUM, 2016).
Following research from the Centre Block BIM, it was determined that a tolerance of less than $25 \mathrm{~mm}$ of modelled elements to point cloud data was suitable for the Library BIM. In a similar methodology, any deviations greater than $25 \mathrm{~mm}$ would be captured within the geometric representation of the building element and any deviations worth noting would be documented within the properties of an individual model element.

\subsection{Data Sources and Management}

The digitization program for the Library was limited in scope. Only the Main Reading Room, stairwells, attic, a few typical offices, and exterior were surveyed to produce geo-referenced point cloud data (Figure 6). We also requested highly detailed meshes of individual heritage assets in the main reading room such as the hand-carved wood rosettes and the statue of Queen Victoria - from photogrammetry since they would be required for the planned visualization applications. The data was captured by CIMS in partnership with HCS using a Leica P40 (main reading room) and a Faro Focus (typical offices, staircases, and attic). One of the challenges in digitizing the Main Reading Room was minimizing occlusions due to the room's complex multi-level, circular geometry. The survey of the Main Reading Room required 97 high-resolution scans in order to meet the required LOD and LOA for the BIM - taking approximately five days to complete. The exterior of the Library was captured by HCS using UAV photogrammetry. We also relied on scan data from the Centre Block digitization campaign since the Library data was geo-referenced to the same survey network as Centre Block. 2D CAD record drawings from the recent rehabilitation project (2007-2011) were referenced in cases where point cloud data was not available. However, we found significant discrepancies in instances where both point cloud data and record drawings existed.

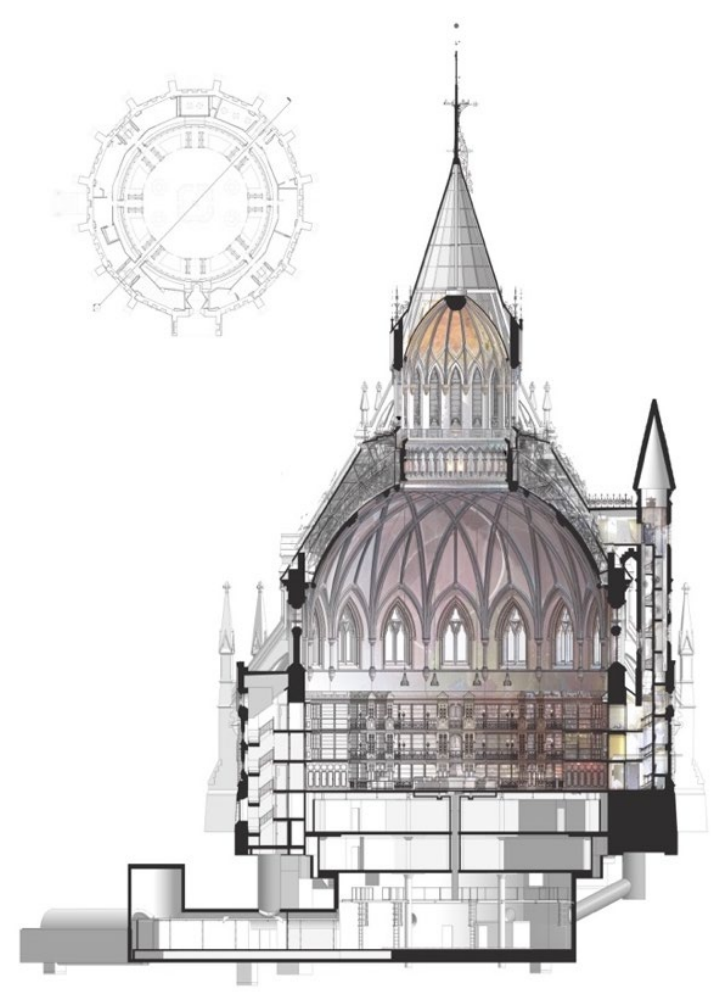

Figure 6: Sectional View of Library of Parliament BIM with coloured point cloud overlay. 
To increase workstation performance while modelling, the .pts files were imported into Autodesk Recap and divided into .rcp files by areas per floor. An exception to this was the Main Reading Room where the 97 .rcs files were imported into Revit as individual scan station locations. The geometry of the room and file size of each scan made it difficult to group the scans into an effective and manageable .rcp file. From our previous research, we were confident that through proper Revit workset management we could contain the whole Library of Parliament building at a high LOD within a single Revit file. This eliminated the inefficiency of switching between models in order to adjust model elements - especially at the join conditions where linked files connected.

\subsection{Modelling Methodology}

Similar in methodology to both the Centre Block and West Block BIM, the first step in modelling Centre Block was integrating the geo-referenced point cloud data into Revit by linking the individual .rep and .res files. The point cloud data was then viewed in a series of $2 \mathrm{D}$ section, elevation, and plan views to trace the profiles of the geometry of the building elements to the appropriate LOD and LOA. Next, using Revit's modelling tools, solid 3D model elements were developed into parametric families. Despite our experience in modelling complex existing conditions, the geometry and detail of the Library - including curved windows, elaborate fixtures, and flying buttresses proved to be an extraordinary challenge (Figure 7).

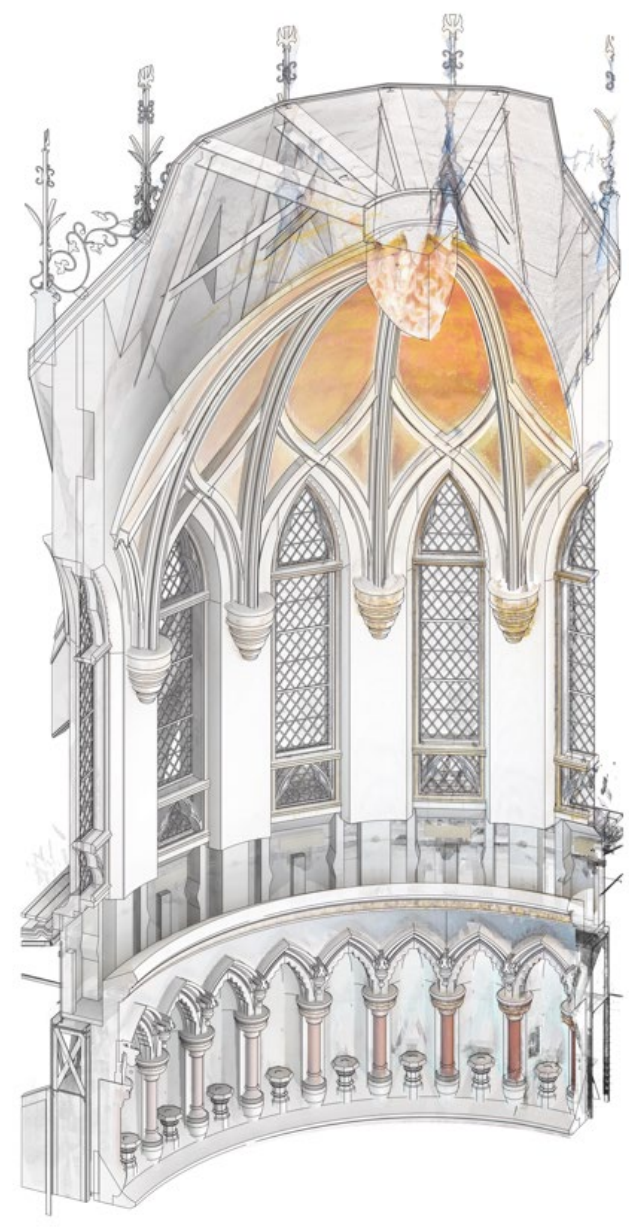

Figure 7: Sectional View of Library of Parliament BIM Lantern with coloured point cloud overlay.
Modelling double curved geometry, intricate details, and surface deformations to a high level of detail from point cloud data required a return to exploring the possibilities of model-in-place families in special situations. For example, the geometry of the domed ceiling of the Main Reading Room was extremely irregular. Our workflow involved producing a conceptual mass family generated directly from point cloud, then applying a generic model ceiling family, and deleting the conceptual mass.

For some parametric families — such as the bookshelves - we utilized custom on/off visibility parameters to hide or show hosted families. For instance, a bookshelf within Stack A is similar to Stack B except for the distinct wooden ornament. Both types of wooden ornament would be hosted within the family, but the option to hide or show either option was available.

\subsection{Results}

Our earlier research on Parliament Hill allowed us to evaluate the trade-offs between LOD and BIM performance, communicate data sources effectively, and use our existing protocols for modelling workflows outlining step-by-step instructions for modelling building elements from point cloud data. However, the increased complexity of the building required the augmentation of our existing protocols and development of novel ones to capture the LOD and LOA required for the specific BIM uses. This resulted in the Library of Parliament Model being our most complex BIM project to date.

\section{QUALITY ASSURANCE}

Initially, the quality assurance methods to evaluate LOA were manual (Marcher et al., 2019). The process for verifying a model created from point cloud data involved creating multiple sectional views along elements in Revit and measuring the deviations that appeared to be the greatest between the point cloud and the model element. This method was time-consuming - notably for large BIM projects — and it limited the verification of the model to specific section locations.

In the summer of 2018, a plug-in for Revit - 3D Analysis - was developed at CIMS (in association with the Photogrammetry and Geometrics Group, INSA). The plug-in is a first step toward an automated visualization of the deviations between Revit wall elements and adjacent point cloud data in a 3D view. After minimal computation time, points are colourized according to the computed deviations and a 3D colour map is displayed. To help the user analyse the deviations, a window containing information about the repartition of deviations is also displayed. The plug-in is proving more efficient in relation to the previous LOA verification processes. Moreover, deviations are represented in a $3 \mathrm{D}$ view making the identification of potential modelling errors and deviations more visible (Marcher et al., 2019)

Although we have achieved some success in automating the point cloud to BIM process, it must be acknowledged that the manual process used for the development of the Parliament Hill BIM has resulted in highly detailed and accurate models. Further, not all information for modelling an historic building is born digital. By synthesizing sensor data with secondary sources such as historical drawings, we have been able to generate a comprehensive representation of the fabric of the building. For example, the structural steel in Centre Block that is hidden from view within walls and floor slabs is now visible in the model and can be understood contextually. These secondary sources integrated into the properties of the model elements for all three 
models - also offer the beginning of a rich database of nongeometrical cultural information related to the construction of the building (eg. steel catalogues).

As we develop LODIA workflows that produce more efficient and geometrically rich models, we continue to augment existing and create new protocols. The implementation of these protocols in the lab ensures consistency across all modelled elements in terms of modelling methodology.

\section{CONCLUSION}

One of the greatest challenges in developing BIM for historical buildings has been in determining the appropriate LOD and LOA. When CIMS began our research partnership with PSPC, we questioned whether BIM - conceived and developed to increase efficiencies for new construction - was the appropriate method for recording the intricate geometries and heritage values of the century-old neo-Gothic Parliament buildings. As our modelling practices evolved from West Block to the Library of Parliament, we were able to produce more efficient and richer models targeted at specific model uses identified by our partners. This confirmed our early hypothesis about the potential of BIM for architectural rehabilitation and conservation. (Fai et.al. 2011) Our ongoing work has demonstrated that BIM can not only serve as an accurate and detailed record of construction, but - through effective data management and visualization - can also serve as an archive for historical information and a window to the palpable sense of heritage these buildings inspire. These are significant considerations for a site that will not be fully accessible to the public for the duration of the rehabilitation project.

To conclude, over the past seven years, our research partnerships with PSPC and - more recently - CENTRUS at the Parliament Hill National Historic Site have continued to expand the boundaries of digital workflows for architectural rehabilitation and heritage conservation. Our ongoing work on LODIA is not intended as an attempt to develop an industry standard. Rather, we see it as forum for discourse and consensus building with our partners in a rapidly evolving field of research. Our intention in this paper is to demonstrate to our public and private partners and to academic colleagues the value added for all parties in applied, collaborative research.

\section{ACKNOWLEDGEMENTS}

CIMS wishes to thank the Parliamentary Precinct Branch, Public Services and Procurement Canada for their ongoing support of our research. Special thanks to Heritage Conservation Services, Real Property Branch, Public Services and Procurement Canada for their technical support. This project was funded in part by the Social Sciences and Humanities Research Council (SSHRC) of Canada through the New Paradigm New Tools for Heritage Conservation in Canada internship program.

\section{REFERENCES}

BIMForum 2013. Level of Development Specification: For Building Information Models, Version: 2013.

BIMForum 2016. Level of Development Specification: For Building Information Models, Version: 2016.

Brumana, R., Oreni, D., Raimondi, A., Georgopoulos, A., Bregianni, A. From survey to HBIM for documentation, dissemination and management of built heritage: The case study of St. Maria in Scaria d'Intelvi. Digit. Heritage Int. Congr. 2013, 1, 497-504.

CanBIM AEC (CAN) Designers Committee, 2012. AEC (CAN) BIM Protocol: Implementing Canadian BIM Standards for the Architectural, Engineering and Construction industry based on international collaboration, V 1.0.

Dore, C., Murphy, M. 2017. Current state of the art historic building information modelling. 42(2) 185-192. doi:10.5194/isprs-archives-XLII-2-W5-185-2017

D’Auria, S., De Feo, E., Di Ruocco, G. Parametric planning for the restoration and rehabilitation of architectural heritage. CSECity Saf. Energy 2014, 2, 107-118.

Fai, S., Rafeiro, J. 2014, "Establishing an Appropriate Level of Detail (LoD) for a Building Information Model (BIM) - West Block, Parliament Hill, Ottawa, Canada", ISPRS Annals of Photogrammetry, Remote Sensing and Spatial Information Sciences, vol. II-5, pp. 123-130

Fai, S., Graham, K., Duckworth, T., Wood, N., Attar, R., 2011. Building Information Modelling and Heritage Documentation. 23rd International Symposium, International Scientific Committee for Documentation of Cultural Heritage (CIPA).

Fai, S., Sydor, M. Building Information Modelling and the documentation of architectural heritage: Between the 'typical' and the 'specific'. Digit. Heritage Int. Congr. 2013, 1, 731-734.

"Federal Heritage Buildings Review Office." Parks Canada, Government of Canada, https://www.pc.gc.ca/en/culture/beefpfhbro (Accessed February 18, 2019).

Graham, K., Chow, L., Fai, S. 2018. Level of detail, information and accuracy in building information modelling of existing and heritage buildings. Journal of Cultural Heritage Management and Sustainable Development. 8. 495-507. 10.1108/JCHMSD-092018-0067.

Hichri, N., Stefani, C., Luca, L. D., Veron, P. Review of the AsBuilt BIM Approaches, 5th International Workshop, 3D-ARCH2013. 3D Virtual Reconstruction and Visualisation of Complex Architectures, Trento, Italy, 107-112.

Historic England 2017, BIM for Heritage: Developing a Historic Building Information Model. Swindon. Historic England.

López, F.J., Lerones, P.M., Llamas, J., Gómez-García-Bermejo, J. \& Zalama, E. 2018, A Review of Heritage Building Information Modeling (H-BIM), Multimodal Technologies and Interaction, vol. 2, no. 2.

Macher, H., Chow, L. Fai, S. 2019, "Automating The Verification Of Heritage Building Information Models Created From Point Cloud Data", The International Archives of the Photogrammetry, Remote Sensing and Spatial Information Sciences, vol. XLII-2-W9, pp. 455-460.

Plowman Craven, 2015. BIM Survey Specification and Reference Guide.

Volk, R., Stengel, J. \& Schultmann, F. 2014, "Building Information Modeling (BIM) for existing buildings - Literature review and future needs", Automation in Construction, vol. 38, 109-127. 